\title{
Compensation for channel dispersion by nonlinear optical phase conjugation
}

\author{
Amnon Yariv, Dan Fekete, and David M. Pepper \\ California Institute of Technology, Pasadena, California 91125
}

Received October 2, 1978

\begin{abstract}
It is proposed that the process of nonlinear optical phase conjugation can be utilized to compensate for channel dispersion and hence to correct for temporal pulse broadening. Specifically, a four-wave nonlinear interaction is shown to achieve pulse renarrowing. Spectral bandwidth constraints of the input pulse are presented for typical phase-conjugate interaction parameters.
\end{abstract}

Recent theoretical ${ }^{1-6}$ and experimental ${ }^{1,5,7-10}$ studies indicate that the generation of phase-conjugate replicas of incident optical waves has certain formal aspects of time reversal and that this property can be used to correct for spatial-propagation distortion. In addition, chirp compensation using four-wave mixing has been analyzed.11

In this Letter we explore theoretically what happens following conjugation to a short electromagnetic pulse that has traversed a dispersive channel. We find, reassuringly, that the group delay of the pulses is not time reversed, i.e., pulses retain their relative temporal order. The effect of group velocity dispersion, $\mathrm{d} v_{g} / \mathrm{d} \omega$, however, is time reversed to first order. This implies that pulses broadened in propagation can be renarrowed following conjugation by merely propagating through a second channel. We remark here that the notion of equalization or estimation and chirp techniques as applied to phase compensation due to pulse propagation is a well-known concept in data communication systems and in radar applications. ${ }^{12}$ The application of nonlinear optical techniques with respect to this problem has not, to our knowledge, been analyzed and is the topic of this Letter.

The model analyzed is shown in Fig. 1. An input pulse,

$$
f_{1}(\mathrm{t})=g(t) \exp \left(i \omega_{0} t\right),
$$

is incident on a dispersive channel. $\omega_{0}$ is the optical carrier frequency; the pulse envelope is $g(t)$. The Fourier transform of $g(t)$ is $F(\Omega)$

$$
g(t)=\int_{-\infty}^{\infty} F(\Omega) \exp (i \Omega t \mathrm{~d} \Omega)
$$

so that

$$
f_{1}(t)=\int_{-\infty}^{\infty} F(\Omega) \exp \left[i\left(\omega_{0}+\Omega\right) t\right] \mathrm{d} \Omega .
$$

Since the width of $g(t)$ is very large compared with the optical period $2 \pi / \omega_{0}$, it follows that $\Omega \ll \omega_{0}$ over the region where $F(\Omega)$ is appreciable.

The propagation constant through the channel is $\beta(\omega)$, so that the output pulse $f_{2}(t)$ is

$$
\begin{aligned}
& f_{2}(t)= \\
& \quad \int_{-\infty}^{\infty} F(\Omega) \exp \left\{i\left[\left(\omega_{0}+\Omega\right) t-\beta\left(\omega_{0}+\Omega\right) L_{1}\right]\right\} \mathrm{d} \Omega,
\end{aligned}
$$

where $L_{1}$ is the length of the channel.

Expanding $\beta(\omega)$ in a Taylor series near $\omega_{0}$,

$$
\beta\left(\omega_{0}+\Omega\right)=\beta\left(\omega_{0}\right)+\frac{\partial \beta}{\partial \omega} \Omega+\frac{1}{2} \frac{\partial^{2} \beta}{\partial \omega^{2}} \Omega^{2}+\ldots,
$$

leads to

$$
\begin{aligned}
f_{2}(t)= & \exp \left[i\left(\omega_{0} t-\beta_{0} L_{1}\right)\right] \int_{-\infty}^{\infty} F(\Omega) \\
& \times \exp \left\{i\left[\Omega\left(t-\frac{\partial \beta}{\partial \omega} L_{1}\right)-\frac{1}{2} \frac{\partial^{2} \beta}{\partial \omega^{2}} \Omega^{2} L_{1}\right]\right\} d \Omega,
\end{aligned}
$$

where $\beta_{0} \equiv \beta\left(\omega_{0}\right)$. The term $(\partial \beta / \partial \omega) L_{1}$ can be written as $L_{1} / v_{g}$, where $v_{g}=\partial \omega / \partial \beta$ is the group velocity. The term $\partial^{2} \beta / \partial \omega^{2}$, which can be written as $\left[-\left(1 / v_{g}{ }^{2}\right)\left(\partial v_{g} /\right.\right.$ $\partial \omega)$ ], is the group velocity dispersion term and causes $f_{2}(t)$ to be broader than $f_{1}(t)$ when $f_{1}(t)$ is a transform-limited pulse.

The pulse $f_{2}(t)$ undergoes phase conjugation-the result being the pulse $f_{3}(t)$. To be specific, we will assume that the phase conjugation is achieved by fourwave mixing in a nondispersive medium with two (essentially cw) pump waves, $A_{1}$ and $A_{2}$, at $\omega_{0}$. We further assume that the response time of the nonlinear interaction is faster than the pulse duration. This causes a Fourier component at $\omega_{0}+\Omega$ to be reflected ${ }^{13}$ at a frequency $\omega_{0}-\Omega$, so that the sum $2 \omega_{0}$ of the pump frequencies is equal to the sum of the incident and reflected frequencies. In general, an incident Fourier component $A \exp \left[i\left(\omega_{0}+\Omega\right) t\right]$ is reflected from the conjugator as $r(\Omega) \exp [i \phi(\Omega)] A^{*} \exp \left[i\left(\omega_{0}-\Omega\right) t\right]$, where $r(\Omega) \exp [i \phi(\Omega)]$ is the complex reflection coefficient of the conjugator ${ }^{13}$ at $\omega_{0}+\Omega(r$ is the magnitude of the reflectivity). The total reflected wave is

$$
\begin{array}{r}
f_{3}(t)=\exp \left[i\left(\omega_{0} t+\beta_{0} L_{1}\right)\right] \\
\times \int_{-\infty}^{\infty} r(\Omega) F^{*}(\Omega) \exp \left\{i \left[-\Omega\left(t-\frac{\partial \beta}{\partial \omega} L_{1}\right)\right.\right. \\
\left.\left.+\frac{1}{2} \frac{\partial^{2} \beta}{\partial \omega^{2}} \Omega^{2} L_{1}+\phi(\Omega)\right]\right\} \mathrm{d} \Omega .
\end{array}
$$




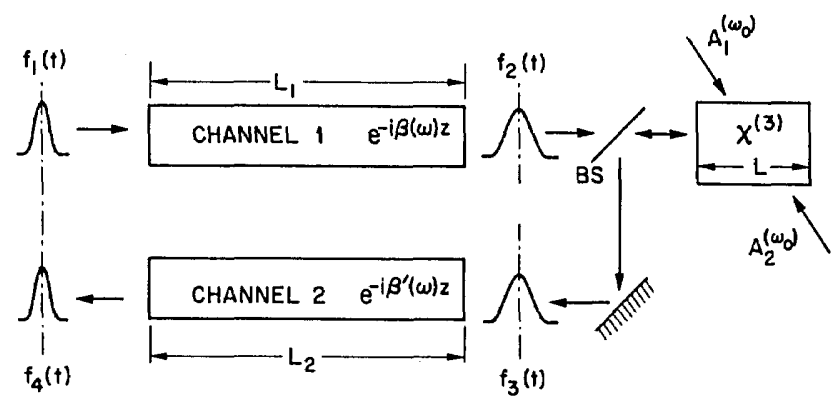

Fig. 1. Schematic diagram of pulse renarrowing scheme. The phase delays of channels 1 and 2 are given by $\beta(\omega)$ and $\beta^{\prime}(\omega)$, respectively. The input pulse spectral envelope of $f_{1}(t)$ is assumed to be transform limited. BS is a beam splitter.

The wave $f_{3}(t)$ is incident on a medium of length $L_{2}$ whose propagation constant at $\omega_{0}-\Omega$ can be expanded as

$$
\beta^{\prime}\left(\omega_{0}-\Omega\right)=\beta_{0}{ }^{\prime}-\frac{\partial \beta^{\prime}}{\partial \omega} \Omega+\frac{1}{2} \frac{\partial^{2} \beta^{\prime}}{\partial \omega^{2}} \Omega^{2}+\ldots
$$

The output $f_{4}(t)$ is given by

$$
\begin{aligned}
f_{4}(t)=\exp & {\left[i\left(\omega_{0} t+\beta_{0} L_{1}-\beta_{0} L_{2}\right)\right] \int_{-\infty}^{\infty} r(\Omega) F^{*}(\Omega) } \\
& \times \exp \left\{i \left[-\Omega\left(t-\frac{\partial \beta}{\partial \omega} L_{1}-\frac{\partial \beta^{\prime}}{\partial \omega} L_{2}\right)\right.\right. \\
& \left.\left.+\frac{1}{2}\left(\frac{\partial^{2} \beta}{\partial \omega^{2}} L_{1}-\frac{\partial^{2} \beta^{\prime}}{\partial \omega^{2}} L_{2}\right) \Omega^{2}+\phi(\Omega)\right]\right\} \mathrm{d} \Omega .
\end{aligned}
$$

The total group delay, $(\partial \beta / \partial \omega) L_{1}+\left(\partial \beta^{\prime} / \partial \omega\right) L_{2}$, is the sum of the individual channel delays. The group velocity dispersion term, which involves the second-order derivatives in Eq. (8), disappears, to first order, if

$$
\frac{\partial^{2} \beta}{\partial \omega^{2}} L_{1}=\frac{\partial^{2} \beta^{\prime}}{\partial \omega^{2}} L_{2}
$$

This requires that the sign of $\partial^{2} \beta / \partial \omega^{2}$ be the same in both channels. At the output of channel 2, the pulse is renarrowed. For maximum renarrowing, it is necessary that $r(\Omega)$ be a constant over the spectral range of the envelope function $F(\Omega)$ and that $\phi(\Omega)=a+b \Omega$, where $a$ and $b$ are two real constants. Under these conditions the output from channel 2 is

$$
\begin{aligned}
& f_{4}(t)=\exp \left[i\left(\omega_{0} t+\beta_{0} L_{1}-\beta_{0} L_{2}+a\right)\right] \int_{-\infty}^{\infty} F^{*}(\Omega) \\
& \quad \times \exp \left\{i\left[-\Omega\left(t-\frac{\partial \beta}{\partial \omega} L_{1}-\frac{\partial \beta^{\prime}}{\partial \omega} L_{2}-b\right)\right]\right\} \mathrm{d} \Omega .
\end{aligned}
$$

Thus,

$$
f_{4}(\mathrm{t})=e^{i \Psi}\left[e^{i \omega_{0} t_{\mathrm{g}} *}\left(t-t_{\mathrm{g}}\right)\right]
$$

where

$$
\Psi=\beta_{0} L_{1}-\beta_{0}{ }^{\prime} L_{2}+a
$$

and

$$
t_{g}=\frac{\partial \beta}{\partial \omega} L_{1}+\frac{\partial \beta^{\prime}}{\partial \omega} L_{2}+b,
$$

which corresponds to the total group delay.
Hence, in addition to conjugation and a reflection of the frequency spectrum from $\omega_{0}+\Omega$ to $\omega_{0}-\Omega$, the four-wave mixing process introduces a group delay of $b$. Under these conditions and neglecting higher orders in expansion (4), the pulse at the output of channel 2 regains its original width.

To check the assumptions mentioned above concerning the phase conjugator, we plot in Fig. 2 the calculated complex reflection coefficient $r(\Omega) \exp [i \phi(\Omega)]$ of a typical conjugator. ${ }^{13}$ We find that, for $\Omega<4 \mathrm{GHz}$, the deviation of $\phi(\Omega)$ from a straight line is very small compared with $\pi$, so that pulses with width of $\gtrsim 2 \times$ $10^{-10}$ sec can be renarrowed by conjugation. Under these conditions, the constants $a$ and $b$ in the expansion of $\phi(\Omega)$ are $\phi_{p}-\pi / 2$ and $n L / c$, respectively, where $L$ and $n$ are the interaction length and linear refractive index, respectively, of the phase conjugator and $\phi_{p}$ is the phase of the pump waves. Thus the additional group delay due to the four-wave interaction is given by $t_{g}{ }^{\prime}=$ $n L / c$.

On the philosophical side, it is interesting to note that, according to Eq. (11), the effect of conjugation plus the flipping of the frequency spectrum is to time-invert the effect of group velocity dispersion and all additional terms in Eq. (4) that are even in powers of $\Omega$, but not the group delay (along with other terms odd in powers of $\Omega$ ). The group delays $(\partial \beta / \partial \omega) L_{1}$ and $\left(\partial \beta^{\prime} / \partial \omega\right) L_{2}$ of the two legs are additive. The application of the term "timeinversion" to the conjugation process should thus be highly qualified.

We note that other nonlinear interactions that involve phase conjugation and frequency-flipping effects, such as three-wave mixing 2,7 [i.e., $\omega_{\text {out }}=2 \omega-(\omega+\Omega)$ ], can also give rise to pulse renarrowing, as described above. An analysis of the complex frequency-dependent transmission coefficient due to a three-wave mixing process process yields a constant phase factor similar to that given above. However, because of the copropagating nature of the input and output fields in a three-wave interaction, the acceptable input frequency spectrum $[F(\Omega)]$ depends primarily on material dis-

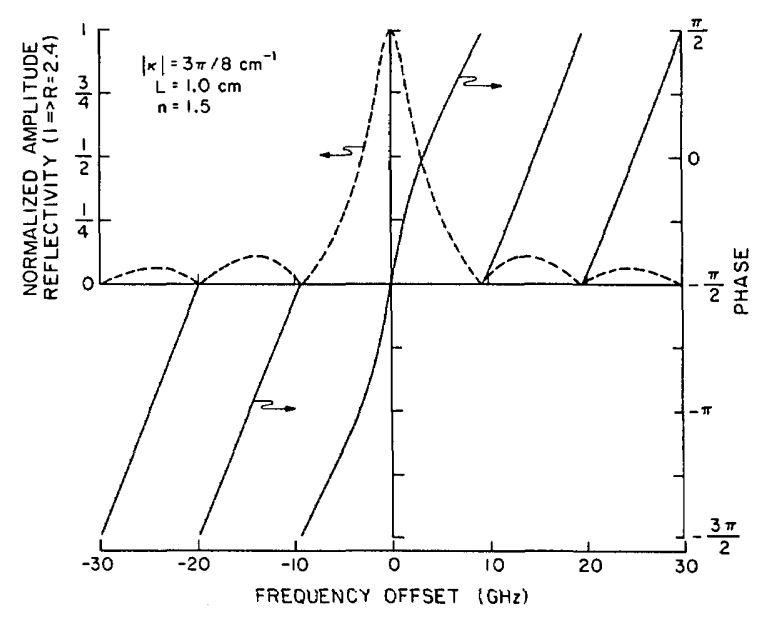

Fig. 2. Magnitude and phase of the complex reflection coefficient versus frequency offset for typical four-wave phase conjugator parameters (after Ref. 13). 
persion, and, to first order in $\Omega$, the medium does not introduce an additional overall group delay. Thus, the acceptable bandwidth for pulse renarrowing may be greater in three-wave versus four-wave interactions. Interactions that yield phase-conjugate fields without frequency flipping (e.g., stimulated Brillouin scattering and stimulated Raman scattering) may also be useful in pulse renarrowing.

In conclusion, we have shown that nonlinear optical conjugation of a dispersion-broadened pulse can be used to obtain a renarrowed pulse by subsequent traversal through a second channel. The input-frequency bandwidth is essentially limited by the phase-matching requirement of the specific nonlinear mixing scheme.

This research was supported by the Air Force Office of Scientific Research. D. Fekete is a Weizmann Institute Postdoctoral Fellow and wishes to acknowledge the support of the Weizmann Institute. D. M. Pepper is a Hughes Research Laboratories Doctoral Fellow and is grateful and thankful for the support granted by the Hughes Aircraft Company.

\section{References}

1. B. Ya. Zel'dovich, V. I. Popovichev, V. V. Ragul'skii, and F. S. Faisullov, JETP Lett. 15, 109 (1972).

2. A. Yariv, Appl. Phys. Lett. 28, 88 (1976).

3. R. W. Hellwarth, J. Opt. Soc. Am. 67, I (1977).

4. A. Yariv and D. M. Pepper, Opt. Lett. 1, 16 (1977).

5. D. M. Bloom and G. C. Bjorklund, Appl. Phys. Lett. 31, 592 (1977).

6. R. L. Abrams and R. C. Lind, Opt. Lett. 2, 94 (1978).

7. P. V. Avizonis, F. A. Hopf, W. D. Bomberger, S. F. Jacobs, A. Tomita, and K. H. Womack, Appl. Phys. Lett. 31, 435 (1977).

8. P. F. Liao and D. M. Bloom, Opt. Lett. 3, 4 (1978), and references therein.

9. S. M. Jensen and R. W. Hellwarth, Appl. Phys. Lett. 32, 166 (1978).

10. D. M. Pepper, D. Fekete, and A. Yariv, Appl. Phys. Lett. 33, 41 (1978).

11. J. H. Marburger, Appl. Phys. Lett. 32, 372 (1978).

12. See, for example, H. Bode, Network Analysis and Feedback Amplifier Design (Van Nostrand, New York, 1945) or F. G. Stremler, Introduction to Communication Systems (Addison-Wesley, Reading, Mass., 1977).

13. D. M. Pepper and R. L. Abrams, Opt. Lett. 3, 212 (1978). 